\title{
Apuntes sobre el Español Dominicano
}

7 UERon recogidos estos datos hace cerca de treinta años en una breve visita a la República Dominicana. Hice una excursión por el sudoeste del país durante la cual pude examinar el habla de cuatro campesinos, jornaleros, analfabetos; uno era natural y vecino de San Cristóbal, cabeza de la actual provincia de Trujillo; otro de Baní, provincia de Trujillo Valdés, y dos de Barro Arriba y Guanábana, provincia de Azua. En una nueva salida hacia el norte utilicé otros tres sujetos de la misma clase social que los anteriores en Jeremías, lugar próximo a La Vega; en Guayacanes, provincia de Santiago, y en Puerto Plata. Un tercer viaje por la parte oriental me dio ocasión para servirme de dos cortadores de caña en el pueblo de San Francisco, provincia del Seibo. En espera de una nueva visita más detenida para completar el trabajo ha transcurrido el tiempo sin publicar estas observaciones.

Entretanto, Pedro Henríquez Ureña, en su libro El español en Santo Domingo, Buenos Aires, Instituto de Filología, 1940, estudió con su habitual maestría varios aspectos de la lengua de su país, especialmente en lo que se refiere al fondo arcaico de su vocabulario y morfología, indicando, además, los rasgos generales de su sistema fonético en el nivel culto y popular. Las presentes notas se limitan a ofrecer una reducida muestra de esta misma lengua en sus manifestaciones propiamente rurales e iletradas. Aunque rápidas e incompletas, podrán servir de base para comprobar las modificaciones que hayan podido producirse a este respecto en el pasado cuarto de siglo $\mathrm{y}$, asimismo, para estimular al futuro 
investigador que tome a su cargo la elaboración del atlas lingüístico dominicano.

VoCAlES ACENTUADAs. La $a$ de timbre medio fue general en palabras como brazo, pala, espada, labio. Los sujetos de Jeremías y San Francisco pronunciaron $a$ marcadamente palatal en vaca, azada y araña. En aire todos los sujetos coincidieron en la variante palatal y, asimismo, en raíces, oído como raíse en San Cristóbal; ráise, en Guanábana y Jeremías, y raíz, pronunciado raí en Bani, rai en Barro y Puerto Plata y rei en San Francisco. No se palatalizó la $a$ en gallo, mayo, calle, saya. La tendencia a la palatalización de esta vocal resultó visiblemente menor que en Puerto Rico. Fue velar la $a$ en baúl, flauta y yautía, y vacilante entre velar y media en bocao y ahogao, así como también ante $j$ aspirada en paja, bajo, etc. No se velarizó en caldo, alto, calvo, ni tampoco ante la aspiración de la $s$ en basto, pasta. En conjunto, el papel de la $a$ velar parece menor en dominicano que en castellano.

La $e$ media se oyó en cabeza, cabello, diente, penca, y la abierta en contacto con $r r$, perro, tierra; delante de $j$ y $s$ aspiradas, abeja, oreja, cesta, pesca; en el diptongo ei, peine, reina, seis, mamey, y en sílaba trabada por $l$, clavel, papel. Aparte de esto, el sujeto de San Francisco hizo abierta la $e$ de bandera, y los de Guanábana y Barro dieron ese mismo timbre a la de teta. La de dedo, pronunciado deo, fue media en Baní, Barro y San Franciseo y abierta en mayor o menor grado en San Cristóbal, Guanábana, Jeremías y Guayacanes. La pronunciación ordinaria de veinte y treinta fue vente y trenta con vacilación entre $e$ media y cerrada en unos sujetos y con $e$ decididamente cerrada en otros. Grados de vacilación entre el timbre medio y el cerrado se observaron también en la $e$ acentuada de leche. La inclinación hacia la $e$ abierta fue, sin duda, mayor que en castellano, aunque no tanto como la observada en Puerto Rico. Hubo labialización relativamente desarrollada en la $e$ del diptongo en nueve, hueso, puente, tuerto; bejuco fue pronunciado casi böuco en San Cristóbal.

La o media se manifestó de manera general en sílaba libre, repollo, retoño, ocho, boca, lechosa, mariposa. Con timbre abierto ocurrió en rosa, hoy, voy ,ojo, hoja, mazorca, jornal flor, sudor, bastón, carbón. Hubo, además, abundantes 
ejemplos de $o$ abierta en casos correspondientes a sílaba libre: la de cola fue abierta en San Francisco y la de joven en Jeremías y Guayacanes; la de toro sólo resultó media en San Cristóbal y Baní; las dos primeras de cogollo, pronunciado cooyo y también cohoyo, con aspiración intermedia, fueron abiertas en Baní, Barro, Jeremías y Puerto Plata y medias en San Cristóbal y San Francisco. Como en el caso de la $e$, la tendencia a la abertura en la $o$ acentuada en sílaba libre parece ser en dominicano más general que en castellano, sin llegar al grado de desarrollo que este fenómeno suele ofrecer en Puerto Rico.

Fueron registradas con timbre medio las vocales $i, u$ en sílaba libre, pico, silla, gallina, nudo, puño, pluma, y con sonido más o menos abierto en sílaba trabada, en contacto con $r r$ y delante de $j$, cincha, risa, hijo, cajuil.

Vocales inacentuadas. Conservan su carácter las vocales inacentuadas sin cambios de consideración en su aspecto articulatorio. Aun en posición final ante pausa, su ordinario debilitamiento llegó rara vez a extremos de ensordecimiento - reducción. La a mantuvo regularmente su timbre medio en yunta, botella, espada, bestia, espiga, abeja, etc. En la pronunciación del sujeto de San Francisco pudo advertirse una clara metafonía en que la a final de vaca mostró el mismo timbre palatal de la acentuada, mientras que la de flauta resultó velar, de acuerdo, asimismo, con el carácter del diptongo anterior. El sujeto de Jeremías dio análogo timbre palatal a las dos vocales de maya; los de San Cristóbal, Guanábana, Barro y Guayacanes palatalizaron igualmente la final de chincha, y los de Guayacanes y San Francisco la de cejas. Sólo en Barro resultó enteramente ensordecida la a de silla; en Guayacanes la de cincha, y en San Francisco la de semilla y rosa.

Conservó la $e$ final el timbre medio, más o menos apagado, en peine, ubre, veinte, etc. Al lado de esta variedad se dieron repetidos ejemplos de $e$ relajada y cerrada. En Baní, Guayacanes y San Francisco apareció esta modificación en nueve, puente, diente y frente. Se observó, sobre todo, tal variedad cerrada en la final de leche, pronunciada ordinariamente con el sonido indicado, a excepción del sujeto de San 
Cristóbal, que la hizo sorda de timbre medio, y del de Barro, que la pronunció con marcada inclinación al tipo abierto.

Por su parte, la o final, dentro de su ordinaria atenuación, mantuvo de ordinario el timbre medio, hueso, palo, estribo, guarapo, banco, seto, etc. El reflejo metafónico se advirtió en la última o de toro, influida por la abertura de la precedente en Barro y Guayacanes. Bajo análogo efecto resultó cerrada de manera general tras de $i$, $u$, acentuadas, pico, potrico, castillo, puño, nudo. En realidad, llegó a aparecer como propia $u$ de timbre abierto la de pico en San Cristóbal, la de castillo en Baní y la de puño en Guayacanes. La palabra nudo, pronunciada, en general, nudu, con débil $d$ fricativa, se redujo a $n u$ en Jeremías y Puerto Plata. Fue corrientemente media la final de cocuyo no obstante las dos uu precedentes en la común pronunciación, cucuyo. Perdió alguna vez la sonoridad en hueso, Bani ; pico, Guayacanes; caballo, San Francisco. Parece que la tendencia a la reducción y ensordecimiento de la vocal inacentuada final es en Santo Domingo menor que en algunas zonas de Puerto Rico, pero es posible que un estudio más completo descubra análoga inclinación en alguna parte del territorio dominicano.

Consonantes B, D, G. Se observa, como en el español normal, la diferenciación entre las modalidades oclusiva y fricativa de las consonantes $b, d, g$. Como rasgo particular, se notó la marcada relajación de la articulación fricativa en varios de los ejemplos registrados. La bilabial se manifestó de manera especialmente tenue y abierta en globo, llave y huevo. La $v$ presentó el mismo sonido bilabial de la $b$. La forma ordinaria de abuelo fue agïelo.

Predominó la eliminación total de la $d$ en patada, azada, desnudo, bocado, dedo, oído; en algunos casos, sin embargo, dentro de estas mismas palabras se advirtió la presencia de la indicada consonante con leve y suave sonido. En nudo, ya citado, la pronunciación con reducida $d$ alternó con las variantes nuo y $n u$. En los sujetos de Jeremías y Guayacanes se recogió narisú, narizudo y, dehnú, desnudo. Viuda con leve $d$ fue más frecuente que viua. El grupo $d r$ se produjo con sonido abreviado y débil en cuadro y piedra. Del mismo modo se oyó en padre y madre en los sujetos de Bani, San Cristóbal y Ba- 
rro. El de Jeremías dijo pae y mae, el de Guanábana pai y mai, y los de Guayacanes y San Francisco taita y mamá. Hubo ausencia completa de la $d$ final en verdad y ciudad, esta última pronunciada suidá.

La articulación blanda y reducida de la $g$ fue notada en fuego, ciego, ahogado, etc. En Baní, San Cristóbal, Barro y San Francisco, la pronunciación de suegro apareció con atenuada articulación del grupo gr; los sujetos de Guanábana, Jeremías y Guayacanes dijeron suedro con la misma atenuación de $d r$. El de Guayacanes decía también adricultura. El cambio inverso $g$ en lugar de $d$, lo hizo el sujeto de San Francisco en ardilla, pronunciado aiguiya.

Fricativas F, S. J. Rara vez se oyó la $f$ como labiodental pura; se mostró de ordinario como articulación mixta bilabiodental en fito, forma, frente y flor. El carácter bilabial se hizo pleno ante la semiconsonante labiovelar en fuego, fuelle, fuerza, fuera, fuimos, fue.

Ninguno de los sujetos examinados aspiró la $h$ en hacer ni en hijo. El de Jeremías aspiró tal consonante en hoja; el de Guayacanes la de harinear, lloviznar, y los de Guanábana, San Francisco, Jeremías, Guayacanes y Puerto Plata la de ahogado. Fue aspirada por todos la de hurón. Según estos ejemplos, la aspiración de la $h$, como en las demás regiones, se mantiene en unas palabras, en otras vacila y en otras desaparece.

La $s$ inicial de sílaba se manifestó uniformemente con articulación predorsal dentoalveolar más o menos convexa. Final de sílaba ante consonante oclusiva sorda experimentó algunas modificaciones. En Baní, Barro y Guayacanes, la $s$ de abispa fue pronunciada con ligera aspiración; los sujetos de San Cristóbal y San Francisco la sustituyeron por una leve geminación de la $p$; los de Guanábana y Jeremías sencillamente la omitieron. Análogas modificaciones ocurrieron en la $s$ de cáscara, cresta, escopeta, espuela y bastón.

Ante consonante sonora, la $s$ final añadió otras variantes. La de desnudo se oyó como débil aspiración sorda en San Francisco y Barro; como tal aspiración visiblemente nasalizada en Jeremías y Guacacanes; como aspiración sonora en Baní; como suave $r$ fricativa en San Cristóbal, y con eliminación 
de todo sonido en Guanábana. La de islita resultó aspirada sorda en Guanábana y Barro, aspirada sonora en Baní y San Cristóbal y mera asimilación a la $l$ siguiente en Guayacanes. La de desbocado se produjo como tenue aspiración sonora en San Francisco, desapareció enteramente en Guanábana, Jeremías y Guayacanes y apareció con aspiración sorda y metátesis, debohcao, en San Cristóbal y Baní.

Como final absoluta, la $s$ quedó enteramente omitida. Fue común y uniforme el seseo de $z$ y $c e, c i$. Las modificaciones de la $z$ final fueron idénticas a las de la $s$. Es seguro que las notas indicadas no agotan los cambios que la $s$ final debe experimentar en Santo Domingo, como en los demás países de la zona antillana.

Como mera aspiración sorda fue pronunciado el sonido correspondiente a $j$ y ge, gi iniciales en joven, julio, gente, giro. En ningún caso apareció la fricación velar de la $j$ castellana. En posición intervocálica, la referida aspiración resultó unas veces sorda y otras sonora en el habla de los mismos individuos $\mathrm{y}$ hasta en la repetición de las mismas palabras. Alternaron sonoridad y sordez en abeja, espejo, oreja, paja, hoja, hijo, ceja y ojo; dominó la sonoridad en cojo y eje y fue general en los indigenismos bija y cajuil. La aspiración sonora se atenuó hasta hacerse imperceptible en el caso ya mencionado de böúco, bejuco.

Liquidas. La articulación de $r$ y $l$ presentó su ordinaria forma ápico-alveolar en posición inicial de sílaba, cara, fiera, palo, cielo. Fue igualmente regular la $l$ interior, plata, clavo. En el grupo $t r$, las dos consonantes fueron débiles y reducidas, con cierto rehilamiento en la fricación de la $r$, cuatro, letro. La $r$ fue simplemente breve y fricativa, sin rehilamiento, en pobre, piedra, cuadro.

Se dio de manera general la igualación de $l$ y $r$ finales de sílaba en interior de vocablo. En el resultado de esta igualación, la $r$ predominó sobre la $l$ en San Cristóbal, Guanábana y Barro, donde se registró una leve $r$ fricativa, tanto en puerta, tuerto, martillo, tarde como en calvo, soldado, espalda, palmito, pulga y caldo. El sujeto de Baní, en la misma zona sudoeste, logró, con cierto esfuerzo, aplicar la $l$ en palmito y calvo, 
pero su manera corriente consistió en un sonido mixto y oscilante entre $l$ y $r$.

A diferencia de los lugares referidos, en Guayacanes, Puerto Plata y San Francisco, ambas consonantes se vocalizaron reduciéndose al sonido $i$, pueita, mueite, tueito, masoica, ehpaida, paimito, soidao, baiba, puiga, saito. El sujeto de Puerto Plata reforzaba la consonante posterior al diptongo imprimiéndole cierto grado de geminación y convirtiendo las Iricativas en oclusivas, pueitta, masoikka, baibba, taidde, etc. En Jeremías, correspondiente a la zona central de La Vega, entre la vocalización del norte y la igualación del sur, el hecho aparecía reducido a la geminación, muette, masolka, tadde, babba, pugga.

No hubo vocalización ni geminación en dulce, pronunciado durse con débil $r$ en Jeremías como en San Cristóbal, Baní y Barro, y simplemente duse en Puerto Plata, Guayacanes y San Francisco. El sujeto de este último punto decía, sin embargo, baisón, barzón. Tampoco se produjo la vocalización entre las varias modificaciones de la $r$ en yerno, pierna, jornal. El sujeto de Baní empleó su ordinario sonido mixto entre $r$ y $l$; el de San Cristóbal hizo en yerno y pierna una débil $r$ fricativa y en jornal dejó oir este mismo sonido visiblemente nasalizado; los de Guanábana, Barro y Jeremías pronunciaron una leve aspiración nasalizada y más o menos sonora; el de Guayacanes empleó en yerno una $r$ fricativa rehilante y nasalizada, en pierna una débil aspiración sorda y en jornal una simple $n$ asimilada a la siguiente; el de San Francisco mostró una breve aspiración sorda y nasal en yerno y pierna y suprimió todo indicio de sonido correspondiente a la $r$ en jornal.

No menos variable fue la pronunciación de la $r$ en virgen, articulada como suave y breve $r$ fricativa por los sujetos de Baní y Barro, como leve aspiración semisonora por los de Guanábana y Jeremías, como aspiración nasalizada y sorda por los de Guayacanes y San Francisco y como mera $n$ velar por el de San Cristóbal. La mayor parte de las variantes señaladas en relación con la $r$ de yerno, virgen y jornal fueron registradas también en el habla popular de Puerto Rico.

Al contrario que en posición interior, la igualación de $l$ y $r$ finales de dicción manifestó preferencia por la primera 
de estas consonantes, fuera de aquellos lugares en que uno y otra se redujeron a $i$. Es ejemplo representativo la palabra sudor, en la que el sujeto de Baní empleó su habitual sonido mixto de $r$ y $l$; los de San Cristóbal, Guanábana y Barro pronunciaron una débil $l$; los de Guayacanes, Puerto Plata y San Francisco vocalizaron el sonido en $i$ y el de Jeremías lo vocalizó en e cerrada y nasal. Las mismas diferencias se observaron en cazador, ruiseñor, flor y zumbador.

En cañaveral y manantial hubo clara división entre Baní, San Cristóbal, Guanábana y Barro que mantuvieron la $l \mathrm{y}$ de otra parte Jeremías, Guayacanes y San Francisco que la sustituyeron por $i$, la cual a veces sonaba con timbre abjerto, casi como $e$. Dentro de esta misma división, los sujetos de Guanábana y Barro nasalizaron la $l$ final de clavel, juntamente con la $e$ acentuada, a la vez que los de Jeremías, Puerto Plata y San Francisco nasalizaban también el diptongo resultante de la vocalización, clavéi. Las modificaciones de la $l$ en baul y miel siguieron líneas análogas a las de clavel.

La $r$ del infinitivo se mantuvo aunque reducida en San Cristóbal y Baní, golpear, bailar, comer; en Guanábana y Barro se oyć como débil $l$; en Guayacanes, Puerto Plata y San Francisco como $i$ y en Jeremías como $e$ cerrada y nasal. Las modificaciones de las finales $l$ y $r$, juntamente con las de la $s$ en esa misma posición constituyen la materia más imprecisa y variable de la pronunciación dominicana.

VIBRANTE RR. No fue corriente la $r r$ con clara y limpia vibración múltiple a la manera castellana. El único sujeto que usó un sonido de esta especie fue el de Guanábana, en especial después de la vocal acentuada, perro, torre, sierra. En San Cristóbal y Barro lo ordinario fue la $\mathrm{rr}$ apicoalveolar fricativa suave. Este sonido ocurrió de modo regular en los mismos tres lugares mencionados en contacto anterior al acento, rien$d a$, carreta, rueda, rosa, reina. En Baní se advertía cierto elemento rehilante al principio de la fricación y en Jeremías y Guayacanes tal elemento se extendía a toda la consonante, cualquiera que fuese su posición en la palabra. Varias veces este sonido se oyó con timbre asibilado y sordo en el sujeto de Jeremías, sobre todo en principio de vocablo, rueda, rosa, risa. El de San Francisco procedía con extraordinaria inse- 
guridad: hizo $r r$ vibrante pura en rosa, reina, perro y guitarra; fricativa suave en herrero y herrería; rehilante sonora en rienda y risa, y uvular semivibrante en rueda, carreta y garrapata. En ningún otro caso se registró la $r r$ con articulación posterior, uvular o velar, tan abundante en el habla puertorriqueña.

Palatales. En la mayor parte de los casos, la pronunciación de la africada ch coincidió con el sonido del español normal. La correspondencia con este sonido, en la proporción de equilibrio con que se oye en Castilla, ocurrió sobre todo en San Cristóbal, Guanábana, Jeremías y San Francisco. No se registraron casos de predominio del elemento fricativo a la manera del granadino y de otras partes de Andalucía. Las excepciones al uso ordinario se dieron precisamente en sentido opuesto a tal dirección. Fueron instrumento de observación sobre este punto las palabras leche, lechuga, cuchara, ocho, cincha y chinche. El sujeto de Baní hizo oir en toda ocasión una ch de fricación relativamente recortada con ventaja de la parte oclusiva, pero sin sensible desviación del efecto común del sonido. En el habla del sujeto de Barros, la oclusión mostraba predominio más notorio sobre la fricación. El de Guayacanes pronunció la ch como una $t$ dorsopalatal, con fricación apenas perceptible. Es probable que esta modalidad de $c h$ esencialmente oclusiva, muy extendida en Puerto Rico, tenga también en Santo Domingo mayor representación que la que resulta de estos breves datos.

Respecto a la $y$, y del mismo modo respecto a la $l l$ identificada constantemente con la $y$, la práctica general ofreció formas análogas a las que dominan en el español corriente. In posición intervocálica, la articulación fue fricativa suave sin rehilamiento y relativamente cerrada, mayo, rayo, cocuyo, gallo, patilla, sello, cogollo, etc. En algunos casos la estrechez lie gó a alcanzar la modalidad africada dentro de la referida posición intervocálica. Desde luego tal modalidad africada, de timbre suave, no rehilante, fue sobretodo frecuente en principio de palabra, yugo, yunta, llave, lluvia. El único sujeto que en estas ocasiones imprimió a la articulación africada cierto grado de rehilamiento fue el de Guanábana. Hubo también considerable número de casos en que la $y$ o $l l$ inicial, en los mismos voca- 
blos mencionados, no fue africada sino fricativa. No se registraron ejemplos de $y$ abierta, relajada o vocalizada en palabras como cuchillo, estrella, etc.

NASALES. La palatal $\tilde{n}$ apareció con plena articulación palatal nasal mojada, araña, puño. Como iniciales de sílaba, la labial $m$ y la alveolar $n$ respondieron a su valor común. La $n$ final de palabra ante pausa, en joven, virgen, carbón, pendón, se pronunció uniformemente con reducida y débil articulación velar. Una gran parte del efecto nasal recaía sobra la vocal de la misma sílaba. La nasalización afectó a las varias modificaciones de la $r$ en yerno y pierna, envolvió las articulaciones de las dos primeras sílabas de enjambre y se extendió a la totalidad de la palabra en jornal y mejor. Fue también parte importante en los cambios fonéticos de la palabra virgen. El sujeto de Jeremías nasalizó además, sin relación con ninguna consonante nasal inmediata, la $e$ resultante de la vocalización de la $r$ en sudor, cazador, llover, bailar. La $m$ sustituyó a la $n$ en míhpero, níspero, Guayacanes, y la $l l$ fue reemplazada por la $\tilde{n}$ en $\tilde{n} a m a r$, llamar, en todos los puntos estudiados.

Division. El rasgo de diferenciación más conocido en el habla local consiste en el tratamiento de $l$ y $r$ finales, que según se ha visto da por resultado articulación mixta de ambos sonidos en el sur y vocalización en el norte, desde el Cibao a Puerto Plata, y en El Seibo al este. Una delimitación precisa de este fenómeno está por hacer. Por otra parte parece que tal división no se reduce a ese solo punto. Entre las mismas zonas indicadas se ha advertido también diferencia respecto al sonido de la $r r$ inicial, generalmente fricativa suave en el sur y con tendencia al rehilamiento en el norte. Varias notas del vocabulario subrayan ese mismo contraste. Por ejemplo, la amapola de jardín, llamada cayena en San Cristóbal y Baní, recibió los nombres de sangre de Cristo en Jeremías y moco de pavo en San Francisco. La fruta del árbol del pan con semilla o grano fue llamada pan de fruta por los sujetos de San Cristóbal, Baní y Barro, pan de pobre por el de Jeremías y aiboxán por el de Puerto Plata. La variedad sin semilla, llamada mapén y guapen en los lugares del sur, recibió el nombre de buen pan en el norte.

En el contraste indicado los nombres de carácter más 
antiguo y local suelen ser los del norte y los más modernos y comunes los del sur. El molino de caña, para los sujetos de San Cristóbal, Baní y Guanábana fue ingenio y para los de Puerto Plata, Guayacanes, Jeremías y San Francisco, trapiche. La lanza del arado para los primeros fue timón y para los segundos broncal, aparte del de San Francisco que la llamó flecha. El cubo de la rueda apareció en San Cristóbal, Baní y Barro con el nombre de manzana y en Jeremías, Guayacanes y Puerto Plata con el de tambor. El cuerno del toro fue entre los sujetos del sur cacho y entre los del norte chifle. El machete del campesino fue para aquéllos machete y para éstos colin. El pie del caballo fue, respectivamente, para unos casco y para los otros uña.

VARIEDAD. Aparte del vulgarismo formal que se manifiesta en evidentes y conocidas deformaciones de las palabras y que la acción de la escuela y de los centros urbanos refrena y corrige, el español dominicano en su nivel popular, a pesar de su aparente uniformidad fonética, debida a la consistencia de las vocales, of rece, a juzgar por los presentes datos, numerosas variantes de pronunciación en que representa papel principal la movible y vacilante calidad de las consonantes finales de las sílabas trabadas. En la mayor parte de los casos son fáciles de apreciar las coincidencias de estas modificaciones con las que se observan en los demás países antillanos y en las provincias del sur de España. La causa de sus diferencias de localización dentro del territorio dominicano responde sin duda a circunstancias interiores de la historia local.

De las muestras recogidas del vocabulario así como de las referentes a la pronunciación se deduce que las áreas lingüísticas del país no se limitan a la geografía de la igualación y vocalización de $-l$ y $-r$. Las respuestas a determinados conceptos descubrieron una extraordinaria variedad denominativa repartida por los pueblos. El escarabajo que anida al rededor del tronco de la palma fue llamado catarrón en San Cristóbal y San Francisco, tatarrión en Barro, gongorocho en Balí y abejón de coco en Jeremías y Puerto Plata. A la comejenera se le llamó pan de comején en San Cristóbal y Guanábana, cayo de comején en Baní, panal de comején en Jeremías y San Francisco y casa de comején en Puerto Plata. 
El plátano pequeño de exquisito sabor recibió el nombre de guineo dátil en San Cristóbal, guineo niño en Baní, gustoadentro en Guanábana, guineo de rosa en San Francisco, damemás en Jeremías y guineíto de seda en Puerto Plata. Al plátano de tamaño mayor y de sabor menos delicado se le llamó guineo martinico en San Cristóbal y Guanábana, mampurio, rulo, tresfilos y malpecho en San Francisco, cañohondo en Jeremías y guineo hembra en Puerto Plata. Los nombres dados a la palma real fueron palma real en San Cristóbal, Barro y San Francisco, palma de sigua en Baní, palma de fruta en Guanábana, palma de yagua en Jeremías y palma de puerco en Puerto Plata. El hongo que brota junto a los troncos viejos fue llamado bombón en San Cristóbal, paragüitas y flor de muerto en Baní, yonyón en Guanábana, Barro y San Francisco, flor de tierra en Jeremías y esponja en Puerto Plata.

TrANSCRIPCION. Del conjunto de las observaciones anteriores puede dar idea la transcripción del siguiente relato sobre la confección del pan de yuca, tomado de labios de un jornalero del muelle de Puerto Plata:

Se siembra la yuca de rolito, que es un pedacito de palo como asina, y después que está de provecho, se arranca. Tarda como un año en estar buena. Después que se arranca, se busca un cuchillo y se raspa. Después que se raspa, se busca un guayo y se guaya. Se echa en un saco y se prensa para que salga el agua, y cuando está seca la yuca, se busca un hibo y se cedacea para que salga la fina y se quede arriba la gorda, que le dicen palote. La yuca fina se mete en un burén para que se haga una torta, como de especie de una luna grande. Se pone candela abajo, como entremedio de tres piedras. El burén se pone arriba de las piedras. La torta se pone de un lado y después se voltea para que no se queme, y se saca para dejarse enfriar y poderse comerla o para vender. De la yuca se saca también almidón para almidonar la ropa, y también se hacen panecicos de manera como un bollo, como una cosita cuadrada. Se le echa manteca y anís si se quiere amargo, y si lo quiere dulce, se le echa melado, anís, clavo y más nada. De la yuca se hace hojaldre del mismo almidón, de manera de un pegotico blanco. Son dulces y se hornean como la torta.

TOMÁs NAVARRo, New York. 
se sjémbre la yúke đe daglito 'ké sụm pjasiko a pálo' ko.

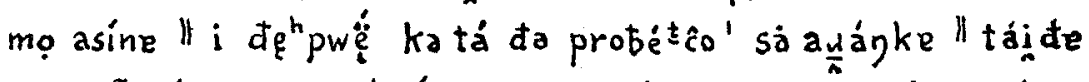

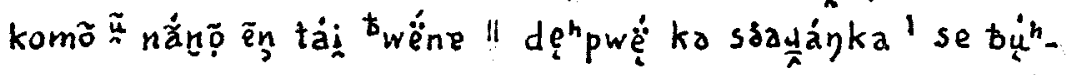

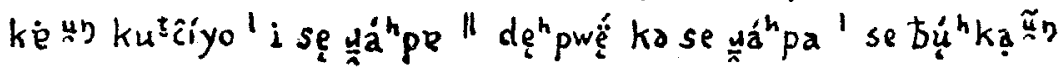
gwáyo' i se qwàye ll sétza a nǘn sáko 'i so aprjénsa' paraka sa sáiga a lágwell i kwando tá séka la yúka ' se tứckạ ${ }^{\tilde{h}} h_{i}$ bo' is a sectaséa 'pa ka sáiga la fina 'i se kéde a a íto la goịđa' ke le disem palóta II la yúka fina sa méte nứm burén' pa ka se ága una tọita komo dệpésje da úna lúna gránda II se póne kandéla táho' komo ontremédjo da trẹ pjẹi.

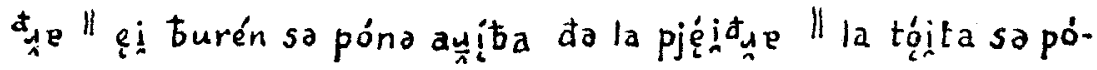
na da unn láo'i đệpwẹ sa tộntéa pare ka nó sa kéma 'i se saka pa đệnäisem friái 'i pođẹiso komẹịya 'o pa beñdẹi" de la yúke so sáke tambjén aimịọ́n 'paimiđonái la dópa' i tambjén so ásem panosiko' de manéra komo un bóyo' komọ una kosita kwađưá ll se létâa mãñtéka yaní si sa kjé to amáigo' i silo kjé đúse' se létca melạo ' aní ' kláboli

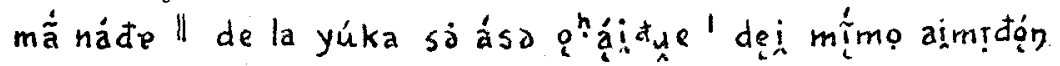

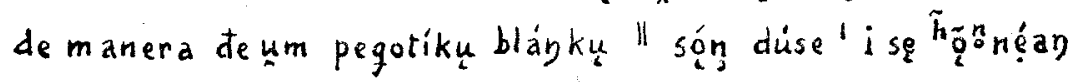
komo la töite. 
